\title{
UTILIZACIÓN DEL OZONO MÉDICO EN EL TRATAMIENTO DEL DOLOR LUMBAR
}

\author{
USE OF MEDICAL OZONE IN THE TREATMENT OF LOW BACK PAIN \\ Urbano Solís ${ }^{(1)}$; Silvia Calvopiña ${ }^{(2)}$. \\ (I) Docente de la Escuela Superior Politécnica de Chimborazo. Riobamba-Ecuador. Docente de la \\ Universidad Nacional de Chimborazo. Riobamba-Ecuador. \\ (2) Empresa de Capacitación Internacional ECI Sc. Riobamba, Ecuador. \\ Email:umsmwork74@gmail.com \\ https://doi.org/10.33789/talentos.7.2.134
}

Resumen: Introducción: El dolor lumbar ha sido señalado como una de las principales causas de invalidez laboral temporal y/o definitiva. Objetivo: Identificar las ventajas de la aplicación de ozono médico como terapia combinada en el tratamiento de pacientes con lumbalgia aguda. Metodología: Se realizó un estudio de casos y controles con dx de lumbalgia aguda en 100 pacientes mayores de 20 años de edad atendidos en la consulta externa de reumatología del Hospital Andino de Chimborazo entre julio 2018 y julio 2019. El grupo control recibió tratamiento con esquemas terapéuticos tradicionales según consensos internacionales para el tratamiento del dolor lumbar agudo. El grupo de casos se adicionó ozono médico, 10 sesiones rectales en tres semanas. Se aplicó la escala visual análoga de dolor y la escala de Oswestry para identificar incapacidad. Se utilizó la prueba de comparación de medias y de McNemar para identificar cambios en la intensidad del dolor, en la presencia y tipo de incapacidad. Resultado: promedio de edad por encima de los 50 años con predominio de pacientes femeninas. La intensidad del dolor al inicio del tratamiento se comportó como media de ambos grupos en 8,76 puntos mientras que el $87 \%$ de los pacientes refirió algún tipo de discapacidad con predominio para la incapacidad moderada. Conclusiones: la aplicación de ozono médico combinado influyó positivamente en la disminución de la intensidad del dolor y en la presencia $y$ tipo de incapacidad.

Palabras clave: Antiinflamatorios; Enfermedad reumática; Lumbalgia; Ozono.

Recibido: 17 de julio de 2020

Aceptado: 19 de octubre de 2020

Publicado como artículo científico en la Revista de Investigación Talentos VII (2), 46-56 
Abstract: Introduction: Low back pain has been indicated as one of the main causes of temporary and / or permanent disability. Objective: To identify the advantages of the application of medical ozone as a combined therapy in the treatment of patients with acute low back pain. Methodology: A case-control study with dx of acute low back pain was carried out in 100 patients older than 20 years of age seen in the rheumatology outpatient clinic of the Andino de Chimborazo Hospital between July 2018 and July 2019. The control group received treatment with schemes Traditional therapies according to international consensus for the treatment of acute low back pain. The group of cases was added medical ozone, 10 rectal sessions in three weeks. The visual analog pain scale and the Oswestry scale were applied to identify disability. The mean comparison test and McNemar's test were used to identify changes in pain intensity, in the presence and type of disability. Result: average age over 50 years with a predominance of female patients. The intensity of pain at the beginning of treatment was 8.76 points as the mean of both groups, while $87 \%$ of the patients referred some type of disability with a predominance of moderate disability. Conclusions: the application of combined medical ozone had a positive influence on the decrease in pain intensity and on the presence and type of disability.

Keywords: Anti-inflammatories; Rheumatic disease; Low back pain, Ozone.

\section{INTRODUCCIÓN}

La lumbalgia aguda es conceptualizada como la presencia de dolor de intensidad variada y de reciente aparición en la región lumbar. De acuerdo al mecanismo etiopatogénico que esté dominando el cuadro se dividen en lumbalgias inflamatorias y lumbalgias mecánicas (Carpio, et al, 2018).

La lumbalgia es considerada por la Organización Mundial de la Salud como una de las 10 primeras causas de morbilidad e incapacidad. Estudios realizados en Cuba describen a las lumbalgias agudas como la tercera causa de incapacidad generando un elevado número de pérdidas de días laborables (Llaguno López, Tino Martínez, \& Álvarez Licona, 2018). En Ecuador, según el Instituto Nacional de Estadísticas y Censos, las lumbalgias agudas constituyen la séptima causa de morbilidad, sin embargo, no existen reportes sobre su influencia en la capacidad funcional de los pacientes (Hernández Arguedas, 2017; Carpio, et al, 2018).

Clínicamente se caracteriza por dolor lumbar de intensidad variable que constituye el principal elemento a tener en cuenta dentro de sus manifestaciones clínicas. Además, el dolor es el principal causante de la incapacidad para poder realizar las actividades de la vida diaria, por lo que ejercer control sobre el dolor constituye el principal objetivo de los esquemas terapéuticos (Solis Cartas, Morejón Gómez, \& de Armas Hernández, 2014; Solis Cartas, Prada Hernández, Morejón Gómez, de Armas Hernandez, \& Amador García, 2015).

En la actualidad el uso de antinflamatorios no esteroideos constituye la piedra angular del tratamiento de la lumbalgia aguda; los esquemas terapéuticos se completan con reposo en estadios iniciales, ejercicios para fortalecer la musculatura lumbar en casos crónicos y/o recurrentes y la aplicación 
de medidas locales; sin embargo, a pesar del uso de los mismos las lumbalgias continúan siendo una enfermedad altamente incapacitante (Lescano Ruiz, Rosero Mera, \& Solís Cartas, 2019).

Recientemente se comienza a utilizar distintas modalidades terapéuticas consideradas como alternativas; dentro de ellas destaca la aplicación de ozono médico de forma combinada con los esquemas terapéuticos habituales. Las propiedades analgésicas y antinflamatorias del ozono médico justifican su aplicación en casos donde primen procesos inflamatorios agudos con diversos grados de intensidad del dolor (Guerrero Guerrero, Miranda Barzagas, Walter Furniel, \& Segura Mora, 2019).

En Ecuador, especialmente en la provincia de Chimborazo y en la ciudad de Riobamba no se cuenta con estudios que abarquen la utilización del ozono médico como terapia combinada en el tratamiento del dolor lumbar. Es por eso que teniendo en cuenta esta situación, la elevada frecuencia con que se presentan las lumbalgias agudas y la afectación que producen a la capacidad funcional y la percepción de calidad de vida relacionada con la salud de los pacientes, se decide realizar esta investigación con el objetivo de identificar las ventajas de la aplicación de ozono médico como terapia combinada en el tratamiento de pacientes con lumbalgia aguda.

\section{MÉTODOS}

Se realizó un estudio aplicado, experimental; donde el universo estuvo constituido por 119 pacientes mayores de 20 años de edad, con diagnóstico confirmado de lumbalgia aguda, según criterios del Colegio Americano de Reumatología, atendidos en la consulta externa de reumatología del Hospital Andino de Chimborazo durante el periodo comprendido entre julio 2018 y julio 2019.

Para realizar el cálculo del tamaño de la muestra se utilizó la fórmula de cálculo muestral para poblaciones conocidas. Se utilizó el método aleatorio simple para conformar la muestra de forma general. La muestra quedó conformada por los 100 pacientes que expresaron, mediante la firma del consentimiento informado, su deseo de participar en el estudio, el cual fue el único criterio de inclusión definido para la investigación.

Con el total de la muestra se confeccionaron dos grupos (control y de casos) cada uno compuesto por 50 pacientes y que dentro de ellos 15 presentaban diagnóstico de lumbalgia inflamatoria y los restantes 35 pacientes de lumbalgia mecánica. De esta forma, se incluyeron en la investigación un total de 70 pacientes con diagnóstico de lumbalgia mecánica y 30 pacientes con lumbalgia inflamatoria. La conformación de los grupos se hizo por muestreo intencionado para lograr una conformación uniforme de ambos.

Antes de comenzar el esquema terapéutico se aplicó en cada paciente tres instrumentos; el primero de ellos fue un cuestionario para identificar características clínicas y generales de los pacientes, este cuestionario fue diseñado específicamente para la investigación y fue sometido al criterio de 7 expertos que en una segunda vuelta manifestaron la opinión favorable para su aplicación. 
El segundo cuestionario utilizado fue la escala visual análoga de dolor (EVAD) que es un instrumento gráfico en el cual los pacientes expresan en una escala de 1 a 10 la intensidad del dolor; siendo 1 la menor intensidad y 10 la mayor intensidad posible del dolor (Santiago Bazán, Perez Domingue, \& Castro Reyes, 2018). El tercer y último cuestionario utilizado fue la escala de Oswestry para determinar incapacidad, esta ha sido validada al español y ampliamente utilizado para determinar presencia y tipo de incapacidad (Moreta Núñez, \& Llumiquinga Pazuña, 2018). La EVAD fue aplicada al empezar el tratamiento, a los 7 días, a los 14 días y al terminar el esquema terapéutico; mientras que la escala de Oswestry se aplicó al inicio y al terminar la aplicación del esquema terapéutico.

Se utilizó como esquema terapéutico en ambos grupos la utilización de diclofenaco sódico en dosis de 150 miligramos diarios durante 21 días, acompañado de 4 gramos diarios de paracetamol los primeros 5 días de tratamiento; en el grupo de casos se adicionó al esquema de ozono terapia que consistió en la administración de 10 sesiones de ozono, a razón de tres sesiones semanales. La primera semana se utilizaron $100 \mathrm{ml}$ de ozono a una dosis de concentración de $15 \mathrm{ug} / \mathrm{ml}$. Durante la segunda semana se aumentó la dosis a 150 $\mathrm{ml}$ de ozono (igual concentración) y durante la tercera y cuarta semana se administraron dosis de $200 \mathrm{ml}$ de ozono.

Se definió el porcentaje de confianza en el $95 \%$, el margen de error en el $5 \%$ y la significación estadística en una $p=0,05$. Se utilizó el programa estadístico SPSS (versión
20,5) para el procesamiento de la información. Se determinaron frecuencias absolutas $y$ porcentajes para variables cualitativas; medidas de tendencia central y de dispersión para variables cuantitativas. Se utilizó la prueba de comparación de medias y la prueba de McNemar para identificar cambios en la intensidad del dolor y en la presencia y tipo de incapacidad. Los resultados se expresaron en forma de tablas para facilitar su comprensión.

Durante el desarrollo de la investigación se cumplieron todas las normas establecidas en la declaración de Helsinki II para realizar investigaciones en seres humanos. Se utilizó el consentimiento informado como documento rector para la incorporación de los pacientes al estudio. La información recopilada fue tratada con total confidencialidad, no se utilizaron datos de identidad personal, solo códigos alfanuméricos y la base de datos con la información recopilada fue destruida después de realizar el informe final de la investigación.

\section{RESULTADOS Y DISCUSIÓN}

Los resultados del presente estudio se muestran a continuación: 
Tabla 1. Distribución de pacientes con lumbalgia aguda según características generales.

\begin{tabular}{|c|c|c|}
\hline \multirow{3}{*}{ Características generales y clínicas } & \multicolumn{2}{|c|}{ Muestra total $n=100$ pacientes con lumbalgia aguda } \\
\hline & $\begin{array}{l}\text { Grupo control } n=50 \\
\text { pacientes }\end{array}$ & $\begin{array}{lll}\text { Grupo } & \text { casos } & \mathbf{n}=\mathbf{5 0} \\
\text { pacientes } & & \\
\end{array}$ \\
\hline & Frecuencia (\%) & Frecuencia (\%) \\
\hline Promedio de edad (años) & 52,56 años DE 27,42 & 51,23 años DE 26,17 \\
\hline \multicolumn{3}{|l|}{ Grupo de edades } \\
\hline Entre 20 y 39 años & $7(14,0)$ & $11(22,0)$ \\
\hline Entre 40 y 59 años & $30(60,0)$ & $27(54,0)$ \\
\hline De 60 años y más & $13(26,0)$ & $12(24,0)$ \\
\hline \multicolumn{3}{|l|}{ Sexo } \\
\hline Masculino & $14(28,0)$ & $9(18,0)$ \\
\hline Femenino & $36(72,0)$ & $41(82,0)$ \\
\hline
\end{tabular}

Fuente: cuestionario de investigación

Elaborado por: Solís Cartas, \& Calvopiña Bejarano, 2020.

Al analizar las características generales de los pacientes incluidos en la investigación (tabla 1) se observó un promedio de edad, en el grupo control, de 52,56 años de edad, con predominio de pacientes entre 40 y 59 años de edad (30 casos y 60,0 \%). En el grupo casos el promedio de edad fue ligeramente inferior
(51,23 años) e igualmente predominaron los pacientes entre 40 y 59 años con un total de 27 casos que representa el 54,0\% de los pacientes investigados. En ambos grupos predominaron los pacientes del sexo femenino, con mayor porcentaje en el grupo de casos donde se identificaron 41 féminas $(82,0 \%)$.

Tabla 2. Distribución de pacientes según intensidad del dolor (EVAD)

\begin{tabular}{llc}
\hline & \multicolumn{2}{l}{ Muestra total $\mathbf{n = 1 0 0}$ pacientes con lumbalgia aguda } \\
\cline { 2 - 3 } Intensidad del dolor según EVAD & $\begin{array}{l}\text { Grupo control } \mathbf{n = 5 0} \\
\text { cientes }\end{array}$ & $\begin{array}{l}\text { Grupo casos } \mathbf{n = 5 0} \text { pa- } \\
\text { cientes }\end{array}$ \\
Inicio del tratamiento & 8,73 & 8.79 \\
7 días de tratamiento & 7,02 & 6,61 \\
14 días de tratamiento & 5,03 & 3,24 \\
Terminar el tratamiento & 3,71 & 1,03 \\
\hline
\end{tabular}

Fuente: Cuestionario de investigación

Elaborado por: Solís Cartas, \& Calvopiña Bejarano, 2020.

En la tabla número 2 se muestra el comportamiento de la intensidad del dolor en ambos grupos de pacientes. Al analizar las medias del dolor se observa, en el grupo control un elevado $8,73 \%$ al inicio del tratamiento; valores que fueron disminuyendo a 7,02; 5,03 y 3,71 a los 7 días, 14 días y terminar el tratamiento respectivamente.

En el grupo de casos existió un ligero aumento de la media de intensidad del dolor al inicio del tratamiento $(8,79)$; sin embargo, la disminución fue mayor a medida que trascurrido la investigación. A los 7 días de tratamiento se obtuvo una media de 6,61, 
mientras que a los 14 días la media disminuyó hasta 3,24. Al finalizar el tratamiento los pacientes refirieron puntuaciones bajas que permitieron obtener una media de 1,03 .

Tabla 3. Distribución de pacientes según presencia y tipo de incapacidad al inicio del tratamiento.

\begin{tabular}{lll}
\hline & $\begin{array}{l}\text { Muestra total } \mathbf{n = 1 0 0} \text { pacientes con } \\
\text { lumbalgia aguda }\end{array}$ \\
\cline { 2 - 3 } Presencia y tipo de incapacidad & $\begin{array}{l}\text { Grupo control } \mathbf{n}=\mathbf{5 0} \\
\text { pacientes }\end{array}$ & $\begin{array}{l}\text { Grupo casos } \mathbf{n = 5 0} \\
\text { pacientes }\end{array}$ \\
\cline { 2 - 3 } & Frecuencia $\mathbf{( \% )}$ & Frecuencia $(\mathbf{\%})$ \\
\hline Sin incapacidad & $7(14,0)$ & $6(12,0)$ \\
Con incapacidad & $43(86,0)$ & $44(88,0)$ \\
\hline Tipo de incapacidad & $\mathbf{n = 4 3}$ & $\mathbf{n}=\mathbf{4 4}$ \\
\hline Incapacidad ligera & $11(25,58)$ & $9(20,46)$ \\
Incapacidad moderada & $27(62,79)$ & $30(68,18)$ \\
Incapacidad severa & $5(11,63)$ & $5(11,36)$ \\
\hline
\end{tabular}

Fuente: cuestionario de investigación

Elaborado por: Solís Cartas, \& Calvopiña Bejarano, 2020.

La determinación de la capacidad funcional al inicio de la investigación (tabla 3) mostró un predominio de pacientes con incapacidad en ambos grupos; $86,0 \%$ en el grupo control y $88,0 \%$ en el grupo casos. En el grupo control predominaron los pacientes con incapacidad moderada (27 casos y 62,79\%), seguido de la incapacidad ligera (11 pacientes y 25,58 $\%)$. En el grupo casos también predominaron los pacientes con incapacidad moderada (30 casos y 68,18\%) seguido de casos con incapacidad ligera ( 9 pacientes y 20,46\%). En ambos grupos existieron 5 pacientes $(11,63 \%)$ con incapacidad severa.

Tabla 4. Distribución de pacientes según presencia y tipo de incapacidad al terminar del tratamiento.

\begin{tabular}{|c|c|c|c|}
\hline \multirow{3}{*}{ Presencia y tipo de incapacidad } & \multicolumn{3}{|c|}{ Muestra total $n=100$ pacientes con lumbalgia aguda } \\
\hline & $\begin{array}{l}\text { Grupo control } \mathbf{n}=\mathbf{5 0} \\
\text { pacientes }\end{array}$ & $\begin{array}{l}\text { Grupo casos } \\
\text { pacientes }\end{array}$ & $\mathrm{n}=\mathbf{5 0}$ \\
\hline & Frecuencia (\%) & Frecuencia (\%) & \\
\hline Sin incapacidad & $33(66,0)$ & $46(92,0)$ & \\
\hline Con incapacidad & $17(34,0)$ & $4(8,0)$ & \\
\hline Tipo de incapacidad & $\mathrm{n}=\mathbf{1 7}$ & $\mathrm{n}=7$ & \\
\hline Incapacidad ligera & $10(58,82)$ & $4(100,0)$ & \\
\hline Incapacidad moderada & $6(35,30)$ & $0(0,00)$ & \\
\hline Incapacidad severa & $1(5,88)$ & $0(0,00)$ & \\
\hline
\end{tabular}

Fuente: Cuestionario de investigación

Elaborado por: Solís Cartas, \& Calvopiña Bejarano, 2020.

En la tabla 4 se muestra que al terminar el esquema terapéutico en ambos grupos existió un predominio de pacientes sin incapacidad; sin embargo, los porcentajes de pacientes con incapacidad fueron mayores en los pacientes del grupo control (sin tratamiento con ozonoterapia) con 17 casos y 34,0 \%. En los pacientes del grupo de casos el porcentaje 
de incapacidad fue de solo el $8,0 \%$ ya que 4 al terminar el esquema terapéutico. personas mantenían algún tipo de incapacidad

Tabla 5. Resultados de la prueba de McNemar.

Muestra total $n=100$ pacientes con diagnóstico de lumbalgia aguda

Intensidad del dolor

\begin{tabular}{cc}
\hline $\begin{array}{c}\text { Grupo control } n=50 \\
\text { pacientes }\end{array}$ & Grupo casos $n=50$ \\
pedia $(p)$ & Media $(p)$ \\
\hline
\end{tabular}

Intensidad del dolor al inicio del tratamiento

$0,043 \quad 0,058$

Intensidad del dolor al terminar el tratamiento

$0,011 \quad 0,046$

$0,062 \quad 0,068$

del tratamiento

$0,014 \quad 0,061$

del tratamiento

$\mathrm{Z}=-3,06 \quad \mathrm{p}=0,02$

Fuente: cuestionario de investigación

Al analizar los resultados de la prueba de McNemar para comparar la significación estadística de las variables intensidad del dolor y presencia de incapacidad al inicio y final de la investigación se obtuvo un valor $Z=$ = 3,06 y una $p=0,02$. Estos resultados permiten afirmar que existen evidencias significativas para plantear que la aplicación de ozono médico como tratamiento combinado produjo cambios significativos en la disminución de la intensidad del dolor y en la aparición de incapacidad en los pacientes con lumbalgia aguda

\section{Discusión}

Los cuadros de lumbalgia aguda son considerados como una de las principales afecciones que generan incapacidad y ausentismo laboral. Su elevada frecuencia de aparición y las complicaciones que pueden ocasionar a la capacidad funcional y percepción de calidad de vida de los pacientes, los convierten en un importante problema de salud mundial que merece la atención de especialistas e investigadores (Solis Cartas, Morejón Gómez, \& de Armas Hernández, 2014; Llaguno López, Tino Martínez, \& Álvarez Licona, 2018).

En esta investigación el promedio de edad de ambos grupos estuvo por encima de los 50 años de edad con predominio de pacientes entre 40 y 59 años; sin embargo, llama la atención porcientos elevados de pacientes menores de 40 años que hacen pensar en causas inflamatorias de dolor lumbar; lo que ensombrece más aún la evolución y pronóstico de estos pacientes. Aunque no pudo ser comparado con otras investigaciones debido a que en la mayoría se estudian solamente la lumbalgia mecánica o la inflamatoria, sin incluir pacientes con ambas formas 
etiopatogénicas de la enfermedad (Veloz Velín, 2019).

Un resultado llamativo estuvo representado por el predominio de pacientes femeninas en ambos grupos; es sorprendente que se asocia la lumbalgia inflamatoria con una mayor prevalencia en el sexo masculino (Solis Cartas, Morejón Gómez, \& de Armas Hernández, 2014; Solis Cartas, Prada Hernández, Morejón Gómez, de Armas Hernandez, \& Amador García, 2015) y en el caso de los pacientes con lumbalgia mecánica se asocia a distintos factores de riesgo entre los que destaca la actividad laboral y principalmente agrícola. Resultado que es similar al estudio realizado por Veloz Velín en la provincia Napo (Veloz Velín, 2019).

Precisamente esta puede ser la explicación del predominio de pacientes femeninas en el estudio y es que en el contexto de la investigación la actividad productiva predominante es la agricultura y las féminas son las que se dedican mayormente a cultivar la tierra, dejando al sexo masculino una representación secundaria en la agricultura y dedicándose a otras actividades (Veloz Velín, 2019).

El análisis de los elementos relacionados con la lumbalgia en ambos grupos, antes del comienzo del estudio, mostró una elevada intensidad del dolor; este síntoma ha sido señalado como el más representativo de este tipo de cuadros, constituyendo el principal motivo de incapacidad y de asistencia a consulta. La intensidad del dolor limita considerablemente la deambulación y la realización de actividades de la vida diaria. (Lescano Ruiz, Rosero Mera, \& Solis Cartas,
2019; Veloz Velín, 2019)

Los pacientes que presentaron esquemas terapéuticos tradicionales, basados en la utilización de antinflamatorios no esteroideos (AINEs) presentaron resolución paulatina del cuadro doloroso, pero que incluso a los 21 días después de la utilización de fármacos prescritos todavía presentaba un dolor ligero que podía presentar algún tipo de incapacidad para realizar algunos esfuerzos físicos $\mathrm{y} / \mathrm{o}$ actividades comunes en la agricultura.

Este comportamiento es similar al reportado en la literatura en otras investigaciones relacionadas con el tratamiento de la lumbalgia aguda con AINEs. El bloqueo de las cicloxigenasas disminuye el suministro de ácido araquidónico a nivel hepático y de esta forma disminuye la producción de citosinas pro inflamatorias responsables de la generación del dolor (Veloz Velín, 2019; Benítez Núñez, Gutiérrez Pérez, Torriente O’Farril, \& Mesa González, 2019).

Por su parte los pacientes el grupo de pacientes al cual se le adicionó ozono médico como terapia combinada para el tratamiento de la lumbalgia aguda presentaron una evolución más favorable en relación a la intensidad del dolor; a los 14 días de tratamiento se observan valores más favorables que el grupo control a los 21 días de tratamiento. Al finalizar la aplicación del esquema terapéutico el promedio de la intensidad del dolor se encontraba en valores mínimos que no representa un riesgo para la actividad laboral del paciente ni para su capacidad física para realizar otras actividades.

Las propiedades antinflamatorias del ozono 
médico, dadas por la reducción de los radicales oxidativos que genera disminución del estrés oxidativo y aumento de las reservas de oxígeno en el organismo potencias el efecto antinflamatorio de los AINEs tradicionales. Esta combinación disminuye de forma más rápida la producción de sustancias proinflamatorias favoreciendo la resolución del cuadro doloroso (Valdés Llerena, Velázquez González, \& Aguado Barrena, 2017).

Este resultado es similar el que reporta Valdés Llerena y otros quienes encontraron un alivio más rápido del dolor en los pacientes con dolor lumbar secundarios a alteraciones facetarias (Valdés Llerena, Velázquez González, \& Aguado Barrena, 2017).

En relación a la incapacidad se obtuvo un resultado similar al de otras investigaciones, aumento de la presencia de incapacidad en ambos grupos al inicio de la investigación y con predominio de pacientes con incapacidad moderada (Valdés Llerena, Velázquez González, \& Aguado Barrena, 2017; Ramírez Pozo, \& Montalvo Luna, 2019). Lo que refuerza la afirmación hecha por otros investigadores que catalogan a la lumbalgia aguda como un proceso altamente incapacitante (Benítez Núñez, Gutiérrez Pérez, Torriente O'Farril, \& Mesa González, 2019; Ramírez Pozo, \& Montalvo Luna, 2019).

Después de aplicados los distintos esquemas terapéuticos ambos grupos mostraron disminución considerable de los pacientes con incapacidad y del tipo de incapacidad predominante, resultados que fueron más favorables en los pacientes que recibieron ozono médico como parte del esquema terapéutico. Resultado similar al reportado en la investigación realizada en el Napo (Veloz Velín, 2019).

Este resultado puede ser explicado por la disminución del dolor que experimentaron los pacientes. El dolor constituye el principal factor de incapacidad en los pacientes con lumbalgia aguda; al disminuir la intensidad del mismo disminuye también su capacidad para poder realizar distintos movimientos y actividades; por lo que se deduce que el objetivo primordial en los pacientes con lumbalgia aguda radica en disminuir, a la mayor brevedad posible, el dolor de los pacientes; con esta acción se estaría actuando secundariamente, sobre la incapacidad resultante de esta afección.

Al aplicar la estadística inferencial, mediante la prueba no paramétrica de McNemar se obtuvieron cambios significativos en torno a la disminución de la intensidad del dolor y de la incapacidad que genera los cuadros de lumbalgia aguda, de esta manera se puede afirmar que existe suficiente evidencia para plantear que la aplicación de ozono médico combinada con AINE incide favorablemente en la disminución del dolor y de la incapacidad en los pacientes con lumbalgia aguda.

La limitación fundamental de esta investigación está centrada en la dificultad, en el momento del estudio, de realizar la determinación de determinados componentes de la inflamación que permitirían obtener una mejor significación de las propiedades antinflamatorias del ozono. Sin embargo, a pesar de esto, la investigación proporciona elementos sostenibles para la utilización del 
ozono médico como tratamiento coadyuvante en el tratamiento del dolor lumbar, lo cual puede ser aplicado y generalizado a nivel nacional.

\section{CONCLUSIONES}

Existió un predominio de pacientes femeninas con lumbalgia aguda entre 40 y 59 años de edad. La aplicación de ozono médico combinado influyó positivamente en la disminución de la intensidad del dolor y en la presencia de tipo de incapacidad.

\section{REFERENCIAS BIBLIOGRÁFICAS}

Benítez Núñez, P.P., Gutiérrez Pérez, M., Torriente O'Farril, D., \& Mesa González, M. (2019). Eficacia de la aplicación intradiscal de plasma rico en plaquetas como tratamiento del dolor discogénico lumbar. Investigaciones Medicoquirúrgicas,11(1),[aprox. 9 p.]. Recuperado de: http://revcimeq.sld.cu/ index.php/imq/article/view/450

Carpio, R., Goicochea Lugo, S., Chávez Corrales, J., Santayana Calizaya, N., Collins, J.A., Robles Recalde, J., et al. (2018). Guía de práctica clínica para el diagnóstico y tratamiento de lumbalgia aguda y subaguda en el Seguro Social del Perú (EsSalud). An. Fac. med.,79(4),351-359. Recuperado de: $\quad$ http://www.scielo.org.pe/scielo. php? script $=$ sci_arttext\&pid $=\mathrm{S} 1025$ $\underline{55832018000400014 \& \operatorname{lng}=\mathrm{es}}$

Guerrero Guerrero, Y., Miranda Barzagas, M., Walter Furniel, D., \& Segura Mora,
M. (2019). Tratamiento acupuntural sacrolumbalgia. CMF\# 7. Los Sombreros. MULTIMED,23(3):[aprox. 18 p.]. Recuperado de: http://www. revmultimed.sld.cu/index.php/mtm/ article/view/1218

Hernández Arguedas, F. (2017). Aspectos relevantes desde el punto de vista médico legal en las valoraciones por lumbalgia. Med. leg. Costa Rica,34(2),69-75. Recuperado de: $\quad$ http://www.scielo.sa.cr/scielo. php? $\mathrm{script}=\mathrm{sci}$ arttext\&pid=S1409$\underline{00152017000200069 \& \operatorname{lng}=\mathrm{en}}$

Lescano Ruiz, M.A., Rosero Mera, L.P., \& Solis Cartas, U. (2019). Reporte de necrosis avascular en un paciente con espondilitis anquilosante. Rev Cubana de Reumatolo,21(Supp. 1),[aprox. 8 p.]. Recuperado de: http://www. revreumatologia.sld.cu/index.php/ $\underline{\text { reumatologia/article/view/687 }}$

Llaguno López, R., Tino Martínez, M.J., \& Álvarez Licona, N. (2018). Caracterización sociodemográfica de los pacientes que acudieron a un servicio de rehabilitación de tercer nivel de atención por lumbalgia. Rev. sanid. mil.,73(1),5-9. Recuperado de: $\quad$ http://www.scielo.org.mx/scielo. php? $\mathrm{script}=\mathrm{sci}$ arttext\&pid $=\mathrm{S} 0301$ $\underline{696 \times 2018000100005 \& \operatorname{lng}=\mathrm{es}}$

Moreta Núñez, C.W., \& Llumiquinga Pazuña, E.M. Abordaje osteopático de lumbalgia crónica. Tesis de grado. Universidad Central del Ecuador, Quito, Ecuador. 2018. Recuperado 
de: $\quad$ http://www.dspace.uce.edu.ec/ $\underline{\text { handle/25000/16031 }}$

Ramírez Pozo, E.G., \& Montalvo Luna, M. (2019). Frecuencia de trastornos musculoesqueléticos en los trabajadores de una refinería de Lima, 2017. An. Fac. med.,80(3):337-341. Recuperado de: $\quad$ http://www.scielo.org.pe/scielo. php? script $=$ sci_arttext\&pid $=\mathrm{S} 1025$ $\underline{55832019000300011 \& \operatorname{lng}=\mathrm{es}}$

Santiago Bazán, C., Perez Domingue, K.J., \& Castro Reyes, N.L. (2018). Dolor lumbar y su relacion con el indice de discapacidad en un hospital de rehabilitacion. Rev Cient Cienc Méd,21(2),13-20. Recuperado de: http://www.scielo.org.bo/scielo. php? script $=$ sci_arttext\&pid $=\mathrm{S} 1817$ $\underline{74332018000200003 \& \operatorname{lng}=\mathrm{es}}$

Solís Cartas, U., Morejón Gómez, J.A., \& de Armas Hernández, A. (2014). Ankylosing Spondylitis and Illness of Von Recklinhausen. An uncommon association. Rev Cuba Reumatol.,16(2),240-244. Recuperado de: $\quad$ http://scielo.sld.cu/scielo. php? script $=$ sci_arttext\&pid $=\mathrm{S} 1817$ $\underline{59962014000200010 \& \operatorname{lng}=\mathrm{es}}$

Solís Cartas, U., Prada Hernández, D.M., Morejón Gómez, J.A., de Armas Hernandez, A., \& Amador García, D.M. (2015). Ankylosing Spondylitis and Klippel-Feil Syndrome. An uncommon association. Rev Cuba Reumatol,17(Supl 1),1-11. Recuperado de: $\quad$ http://scielo.sld.cu/scielo. php? script $=$ sci_arttext\&pid $=\mathrm{S} 1817$ -

\section{$\underline{59962015000300004 \& \operatorname{lng}=\mathrm{es}}$}

Valdés Llerena, R., Velázquez González, K., \& Aguado Barrena, O. (2017). Percutaneous treatment with ozone for the treatment of the lumbar facet joint cyst: a case series. Rev cuba anestesiol reanim,16(2),69-75. Recuperado de: $\quad$ http://scielo.sld.cu/scielo. php? script $=$ sci_arttext\&pid $=$ S1726$\underline{67182017000200009 \& \operatorname{lng}=\mathrm{es}}$

Veloz Velín, A.C. Intervención farmacológica para disminuir las complicaciones de lumbalgia aguda, en pacientes adultos jóvenes y medios. Arosemena Tola, Napo. Tesis de posgrado. Escuela Superior Politécnica de Chimborazo, Riobamba, Chimborazo, Ecuador. 2019. Recuperado de: http://dspace.espoch. edu.ec/handle/123456789/12560 